\title{
Derived equivalences for triangular matrix rings
}

\author{
Hiroki Abe and Mitsuo Hoshino
}

\begin{abstract}
We generalize derived equivalences for triangular matrix rings induced by a certain type of classical tilting module introduced by Auslander, Platzeck and Reiten to generalize reflection functors in the representation theory of quivers due to Bernstein, Gelfand and Ponomarev.
\end{abstract}

Let $R$ be a finite dimensional algebra over a field $k$ and $M$ a finitely generated projective right $R$-module. Set

$$
A=\left(\begin{array}{cc}
k & M \\
0 & R
\end{array}\right) \text { and } e=\left(\begin{array}{cc}
0 & 0 \\
0 & 1
\end{array}\right) \in A .
$$

As pointed out by Brenner and Butler (see [?, p.111]), we know from [?] (cf. also [?]) that $\operatorname{Ext}_{A}^{1}(A / A e A, A) \oplus A e \in \operatorname{Mod}-A^{\text {op }}$ is a classical tilting module, i.e., a tilting module of projective dimension at most one (see [?]) with

$$
\operatorname{End}_{A^{\mathrm{op}}}\left(\operatorname{Ext}_{A}^{1}(A / A e A, A) \oplus A e\right)^{\mathrm{op}} \cong\left(\begin{array}{cc}
R & \operatorname{Hom}_{R}(M, R) \\
0 & k
\end{array}\right) .
$$

Our aim is to extend this type of derived equivalence to the case where $M_{R}$ has finite projective dimension. Let $R, S$ be rings and $M$ an $S$-R-bimodule such that $M$ admits a projective resolution $P^{\bullet} \rightarrow M$ in Mod- $R$ with $P^{\bullet} \in \mathcal{K}^{\mathrm{b}}\left(\mathcal{P}_{R}\right)$ and $\operatorname{Ext}_{R}^{i}(M, R)=0$ for $i<d=\operatorname{proj} \operatorname{dim} M_{R}$. Set

$$
A=\left(\begin{array}{cc}
S & M \\
0 & R
\end{array}\right) \quad \text { and } \quad e=\left(\begin{array}{cc}
0 & 0 \\
0 & 1
\end{array}\right) \in A .
$$

We will construct a tilting complex $T^{\bullet} \in \mathcal{K}^{\mathrm{b}}\left(\mathcal{P}_{A}\right)$ associated with $e$ such that

$$
\operatorname{End}_{\mathcal{K}(\operatorname{Mod}-A)}\left(T^{\bullet}\right) \cong\left(\begin{array}{cc}
R & \operatorname{Ext}_{A}^{d}(M, R) \\
0 & S
\end{array}\right)
$$

2000 Mathematics Subject Classification: Primary: 18E30; Secondary: 16G30, 16G70.

Key words: Derived equivalence, Tilting complex, Triangular matrix ring 
(Theorems ?? and ??). Assume further that ${ }_{S} M$ is faithful and that if $d>0$ then $S \stackrel{\sim}{\rightarrow} \operatorname{End}_{R}(M)$ canonically and $\operatorname{Ext}_{R}^{i}(M, M)=0$ for $1 \leq i<d$. Then we will see that

$$
\operatorname{Hom}_{A}^{\bullet}\left(T^{\bullet}, A\right)[d+1] \cong \operatorname{Ext}_{A}^{d+1}(A / A e A, A) \oplus A e
$$

in $\mathcal{D}\left(\operatorname{Mod}-A^{\mathrm{op}}\right)$ and $\operatorname{Ext}_{A}^{d+1}(A / A e A, A) \oplus A e \in \operatorname{Mod}-A^{\text {op }}$ is a tilting module of projective dimension $d+1$ (see [?]) with

$$
\operatorname{End}_{A^{\mathrm{op}}}\left(\operatorname{Ext}_{A}^{d+1}(A / A e A, A) \oplus A e\right)^{\mathrm{op}} \cong\left(\begin{array}{cc}
R & \operatorname{Ext}_{A}^{d}(M, R) \\
0 & S
\end{array}\right)
$$

(Corollary ?? and Remark ??).

For a $\operatorname{ring} A$, we denote by $\operatorname{Mod}-A$ the category of right $A$-modules, by $\bmod -A$ the full subcategory of Mod- $A$ consisting of finitely presented modules and by $\mathcal{P}_{A}$ the full subcategory of $\operatorname{Mod}-A$ consisting of finitely generated projective modules. We denote by $A^{\mathrm{op}}$ the opposite ring of $A$ and consider left $A$-modules as right $A^{\mathrm{op}}$-modules. Sometimes, we use the notation $X_{A}$ (resp., $\left.{ }_{A} X\right)$ to stress that the module $X$ considered is a right (resp., left) $A$-module. We denote by $\mathcal{K}(\operatorname{Mod}-A)(\operatorname{resp} ., \mathcal{D}(\operatorname{Mod}-A))$ the homotopy (resp., derived) category of cochain complexes over Mod- $A$ and by $\mathcal{K}^{\mathrm{b}}\left(\mathcal{P}_{A}\right)$ the full triangulated subcategory of $\mathcal{K}(\operatorname{Mod}-A)$ consisting of bounded complexes over $\mathcal{P}_{A}$. We consider modules as complexes concentrated in degree zero. For any integer $n \in \mathbb{Z}$ we denote by $\mathrm{H}^{n}(-)$ the $n$-th homology and by $(-)[n]$ the $n$-shift of complexes. Also, we use the notation $\operatorname{Hom}^{\bullet}(-,-)$ to denote the single complex associated with the double hom complex. Finally, for an object $X$ in an additive category $\mathfrak{A}$ we denote by add $(X)$ the full subcategory of $\mathfrak{A}$ consisting of direct summands of finite direct sums of copies of $X$.

We refer to [?] for tilting complexes and derived equivalences and to [?], [?] for derived categories.

\section{General case}

Throughout this section, $A$ is a ring and $e \in A$ is an idempotent satisfying the following conditions:

(E1) Ae admits a projective resolution $\varepsilon: P^{\bullet} \rightarrow A e$ in Mod-eAe with $P^{\bullet} \in \mathcal{K}^{\mathrm{b}}\left(\mathcal{P}_{e A e}\right)$, in particular, $d=\operatorname{proj} \operatorname{dim} A e_{e A e}<\infty$;

(E2) $\mu: A e \otimes_{e A e} e A \rightarrow A, x \otimes y \mapsto x y$ is monic;

(E3) $\varphi: e A \rightarrow \operatorname{Hom}_{e A e}(A e, e A e), x \mapsto(y \mapsto x y)$ is monic;

(E4) if $d>0$ then $\varphi$ is an isomorphism and $\operatorname{Ext}_{e A e}^{i}(A e, e A e)=0$ for $1 \leq i<d ;$ and

(E5) $\operatorname{Tor}_{i}^{e A e}(A e, e A)=0$ for $i \neq 0$. 
We define a complex $T^{\bullet} \in \mathcal{K}^{\mathrm{b}}\left(\mathcal{P}_{A}\right)$ as follows: Set $T_{1}^{\bullet}=e A[d+1]$, let $T_{2}^{\bullet}$ be the mapping cone of the composite

$$
\mu \circ\left(\varepsilon \otimes_{e A e} e A\right): P^{\bullet} \otimes_{e A e} e A \rightarrow A e \otimes_{e A e} e A \rightarrow A
$$

and set $T^{\bullet}=T_{1}^{\bullet} \oplus T_{2}^{\bullet}$.

Theorem 1.1. The complex $T^{\bullet} \in \mathcal{K}^{\mathrm{b}}\left(\mathcal{P}_{A}\right)$ is a tilting complex with

$$
\operatorname{End}_{\mathcal{K}(\operatorname{Mod}-A)}\left(T^{\bullet}\right) \cong\left(\begin{array}{cc}
e A e & \operatorname{Ext}_{A}^{d+1}(A / A e A, e A) \\
0 & A / A e A
\end{array}\right) .
$$

Proof. We may assume $P^{i}=0$ unless $-d \leq i \leq 0$. Note that by (E5) we have a projective resolution $P^{\bullet} \otimes_{e A e} e A \rightarrow A e \otimes_{e A e} e A$ in Mod- $A$ with $P^{\bullet} \otimes_{e A e} e A \in$ $\mathcal{K}^{\mathrm{b}}\left(\mathcal{P}_{A}\right)$ and that by $(\mathrm{E} 2)$ we have an exact sequence in $\operatorname{Mod}-A$

$$
0 \rightarrow A e \otimes_{e A e} e A \stackrel{\mu}{\rightarrow} A \rightarrow A / A e A \rightarrow 0 .
$$

Thus $T_{2}^{\bullet}$ is a projective resolution of $A / A e A$ in $\operatorname{Mod}-A$. In particular, $T_{2}^{\bullet} \cong$ $A / A e A$ in $\mathcal{D}(\operatorname{Mod}-A)$.

Claim 1. $\operatorname{Hom}_{\mathcal{K}(\operatorname{Mod}-A)}\left(T_{1}^{\bullet}, T_{1}^{\bullet}[i]\right)=0$ for $i \neq 0$ and $\operatorname{End}_{\mathcal{K}(\operatorname{Mod}-A)}\left(T_{1}^{\bullet}\right) \cong e A e$.

Proof. The first assertion is obvious and $\operatorname{End}_{\mathcal{K}(\operatorname{Mod}-A)}\left(T_{1}^{\bullet}\right) \cong \operatorname{End}_{A}(e A) \cong e A e$.

Claim 2. $\operatorname{Hom}_{\mathcal{K}(\operatorname{Mod}-A)}\left(T_{1}^{\bullet}, T_{2}^{\bullet}[i]\right)=0$ for all $i \in \mathbb{Z}$.

Proof. We have

$$
\begin{aligned}
\operatorname{Hom}_{A}^{\bullet}\left(T_{1}^{\bullet}, T_{2}^{\bullet}\right) & \cong \operatorname{Hom}_{A}^{\bullet}\left(e A, T_{2}^{\bullet}\right)[-d-1] \\
& \cong\left(T_{2}^{\bullet} \otimes_{A} A e\right)[-d-1] .
\end{aligned}
$$

Also, $T_{2}^{\bullet} \otimes_{A} A e$ is isomorphic to the mapping cone of $\varepsilon: P^{\bullet} \rightarrow A e$. Thus $\operatorname{Hom}_{\mathcal{K}(\operatorname{Mod}-A)}\left(T_{1}^{\bullet}, T_{2}^{\bullet}[i]\right) \cong \mathrm{H}^{i}\left(\operatorname{Hom}_{A}^{\bullet}\left(T_{1}^{\bullet}, T_{2}^{\bullet}\right)\right) \cong \mathrm{H}^{i-d-1}\left(T_{2}^{\bullet} \otimes_{A} A e\right)=0$ for all $i \in \mathbb{Z}$.

Claim 3. $\operatorname{Hom}_{\mathcal{K}(\operatorname{Mod}-A)}\left(T_{2}^{\bullet}, T_{1}^{\bullet}[i]\right)=0$ for $i \neq 0$ and $\operatorname{Hom}_{\mathcal{K}(\operatorname{Mod}-A)}\left(T_{2}^{\bullet}, T_{1}^{\bullet}\right) \cong$ $\operatorname{Ext}_{A}^{d+1}(A / A e A, e A)$.

Proof. Note first that

$$
\begin{aligned}
\operatorname{Hom}_{\mathcal{K}(\operatorname{Mod}-A)}\left(T_{2}^{\bullet}, T_{1}^{\bullet}[i]\right) & \cong \mathrm{H}^{i}\left(\operatorname{Hom}_{A}^{\bullet}\left(T_{2}^{\bullet}, T_{1}^{\bullet}\right)\right) \\
& \cong \mathrm{H}^{i}\left(\operatorname{Hom}_{A}^{\bullet}\left(T_{2}^{\bullet}, e A\right)[d+1]\right) \\
& \cong \mathrm{H}^{i+d+1}\left(\operatorname{Hom}_{A}^{\bullet}\left(T_{2}^{\bullet}, e A\right)\right)
\end{aligned}
$$

for all $i \in \mathbb{Z}$. Since $\operatorname{Hom}_{A}^{\bullet}\left(T_{2}^{\bullet}, e A\right)$ is isomorphic to the (-1)-shift of the mapping cone of the composite

$$
\operatorname{Hom}_{A}(\varepsilon, e A e) \circ \varphi: e A \rightarrow \operatorname{Hom}_{e A e}(A e, e A e) \rightarrow \operatorname{Hom}_{e A e}^{\bullet}\left(P^{\bullet}, e A e\right),
$$


by (E3), (E4) we have $\mathrm{H}^{j}\left(\operatorname{Hom}_{A}^{\bullet}\left(T_{2}^{\bullet}, e A\right)\right)=0$ for $j \neq d+1$. Also, since $T_{2}^{\bullet}$ is a projective resolution of $A / A e A, \mathrm{H}^{d+1}\left(\operatorname{Hom}_{A}^{\bullet}\left(T_{2}^{\bullet}, e A\right)\right) \cong \operatorname{Ext}_{A}^{d+1}(A / A e A, e A)$.

Claim 4. $\operatorname{Hom}_{\mathcal{K}(\operatorname{Mod}-A)}\left(T_{2}^{\bullet}, T_{2}^{\bullet}[i]\right)=0$ for $i \neq 0$ and $\operatorname{End}_{\mathcal{K}(\operatorname{Mod}-A)}\left(T_{2}^{\bullet}\right) \cong A / A e A$.

Proof. Since $\operatorname{Hom}_{A}(e A, A / A e A)=0, \operatorname{Hom}_{A}^{\bullet}\left(T_{2}^{\bullet}, A / A e A\right) \cong A / A e A$ as complexes and

$$
\begin{aligned}
\operatorname{Hom}_{\mathcal{K}(\operatorname{Mod}-A)}\left(T_{2}^{\bullet}, T_{2}^{\bullet}[i]\right) & \cong \operatorname{Hom}_{\mathcal{D}(\operatorname{Mod}-A)}\left(T_{2}^{\bullet}, T_{2}^{\bullet}[i]\right) \\
& \cong \operatorname{Hom}_{\mathcal{D}(\text { Mod- } A)}\left(T_{2}^{\bullet}, A / A e A[i]\right) \\
& \cong \operatorname{Hom}_{\mathcal{K}(\operatorname{Mod}-A)}\left(T_{2}^{\bullet}, A / A e A[i]\right) \\
& \cong H^{i}\left(\operatorname{Hom}_{A}^{\bullet}\left(T_{2}^{\bullet}, A / A e A\right)\right) \\
& =0
\end{aligned}
$$

for $i \neq 0$. Also,

$$
\begin{aligned}
\operatorname{End}_{\mathcal{K}(\operatorname{Mod}-A)}\left(T_{2}^{\bullet}\right) & \cong \operatorname{End}_{\mathcal{D}(\operatorname{Mod}-A)}\left(T_{2}^{\bullet}\right) \\
& \cong \operatorname{End}_{\mathcal{D}(\operatorname{Mod}-A)}(A / A e A) \\
& \cong \operatorname{End}_{A}(A / A e A) \\
& \cong A / A e A
\end{aligned}
$$

Now, by the Claims above $\operatorname{Hom}_{\mathcal{K}(\operatorname{Mod}-A)}\left(T^{\bullet}, T^{\bullet}[i]\right)=0$ for $i \neq 0$ and

$$
\operatorname{End}_{\mathcal{K}(\operatorname{Mod}-A)}\left(T^{\bullet}\right) \cong\left(\begin{array}{cc}
e A e & \operatorname{Ext}_{A}^{d+1}(A / A e A, e A) \\
0 & A / A e A
\end{array}\right) .
$$

Next, since $P^{i} \otimes_{e A e} e A \in \operatorname{add}(e A)$ for all $i \in \mathbb{Z}, P^{\bullet} \otimes_{e A e} e A$ belongs to the full triangulated subcategory of $\mathcal{K}^{\mathrm{b}}\left(\mathcal{P}_{A}\right)$ generated by add $\left(T_{1}^{\bullet}\right)$. Then, since we have a distinguished triangle in $\mathcal{K}^{\mathrm{b}}\left(\mathcal{P}_{A}\right)$

$$
P^{\bullet} \otimes_{e A e} e A \rightarrow A \rightarrow T_{2}^{\bullet} \rightarrow,
$$

it follows that $A$ belongs to the full triangulated subcategory of $\mathcal{K}^{\mathrm{b}}\left(\mathcal{P}_{A}\right)$ generated by $\operatorname{add}\left(T^{\bullet}\right)$. Thus $\operatorname{add}\left(T^{\bullet}\right)$ generates $\mathcal{K}^{\mathrm{b}}\left(\mathcal{P}_{A}\right)$ as a triangulated category and $T^{\bullet}$ is a tilting complex.

This finishes the proof of Theorem ??.

Note that we have $\mathrm{H}^{i}\left(\operatorname{Hom}_{A}^{\bullet}\left(T^{\bullet}, A\right)\right) \cong \operatorname{Ext}_{A}^{i}(A / A e A, A)$ for $i \neq d+1$ and $\mathrm{H}^{d+1}\left(\operatorname{Hom}_{A}^{\bullet}\left(T^{\bullet}, A\right)\right) \cong \operatorname{Ext}_{A}^{d+1}(A / A e A, A) \oplus A e$. We consider next the case where $\operatorname{Ext}_{A}^{d+1}(A / A e A, A) \oplus A e \in \operatorname{Mod}-A^{\text {op }}$ is a tilting module (see [?]). Recall that a module is a tilting module if and only if it is isomorphic to a tilting complex in the derived category (see e.g. [?, Proposition 3.9]). Since we have an anti-equivalence of triangulated categories

$$
\operatorname{Hom}_{A}^{\bullet}(-, A): \mathcal{K}^{\mathrm{b}}\left(\mathcal{P}_{A}\right) \stackrel{\sim}{\rightarrow} \mathcal{K}^{\mathrm{b}}\left(\mathcal{P}_{A^{\text {op }}}\right),
$$


$\operatorname{Hom}_{A}^{\bullet}\left(T^{\bullet}, A\right) \in \mathcal{K}^{\mathrm{b}}\left(\mathcal{P}_{A^{\text {op }}}\right)$ is a tilting complex. Thus, if $\operatorname{Ext}_{A}^{i}(A / A e A, A)=0$ for $i \neq d+1$, then

$$
\operatorname{Hom}_{A}^{\bullet}\left(T^{\bullet}, A\right)[d+1] \cong \operatorname{Ext}_{A}^{d+1}(A / A e A, A) \oplus A e
$$

in $\mathcal{D}\left(\operatorname{Mod}-A^{\mathrm{op}}\right)$ and $\operatorname{Ext}_{A}^{d+1}(A / A e A, A) \oplus A e \in \operatorname{Mod}-A^{\text {op }}$ is a tilting module. We denote by $\gamma: A \rightarrow \operatorname{End}_{e A e}(A e), a \mapsto(x \mapsto a x)$ the ring homomorphism given by the left multiplication. Then it is not difficult to see that $\operatorname{Hom}_{A}^{\bullet}\left(T^{\bullet}, A\right)$ is isomorphic to the (-1)-shift of the mapping cone of the composite

$$
\operatorname{Hom}_{A}(\varepsilon, A e) \circ \gamma: A \rightarrow \operatorname{End}_{e A e}(A e) \rightarrow \operatorname{Hom}_{e A e}^{\bullet}\left(P^{\bullet}, A e\right) .
$$

Consequently, we have the following.

Corollary 1.2. Assume that $\gamma$ is injective and that if $d>0$ then $\gamma$ is an isomorphism and $\operatorname{Ext}_{e A e}^{i}(A e, A e)=0$ for $1 \leq i<d$. Then $\operatorname{Ext}_{A}^{i}(A / A e A, A)=0$ for $i \neq d+1$ and $\operatorname{Ext}_{A}^{d+1}(A / A e A, A) \oplus A e \in \operatorname{Mod}-A^{\text {op }}$ is a tilting module with

$$
\operatorname{End}_{A^{\mathrm{op}}}\left(\operatorname{Ext}_{A}^{d+1}(A / A e A, A) \oplus A e\right)^{\mathrm{op}} \cong\left(\begin{array}{cc}
e A e & \operatorname{Ext}_{A}^{d+1}(A / A e A, e A) \\
0 & A / A e A
\end{array}\right) .
$$

Example 1.3. Let $R$ be a commutative ring and $c \in R$ a regular element which is not a unit. Let $n \geq 2$ be an integer and $\left(m_{i j}\right)$ an $n \times n$ matrix of non-negative integers such that $m_{i i}=0$ for all $1 \leq i \leq n$ and $m_{i j}+m_{j k} \geq m_{i k}$ for all $1 \leq i, j, k \leq n$. Let $A$ be the subset of $\mathrm{M}_{n}(R)$, the $n \times n$ full matrix algebra over $R$, consisting of matrices $\left(x_{i j}\right) \in \mathrm{M}_{n}(R)$ with $x_{i j} \in c^{m_{i j}} R$ for all $1 \leq i, j \leq n$ and denote by $e$ the matrix $\left(x_{i j}\right) \in A$ such that $x_{n n}=1$ and $x_{i j}=0$ unless $i=j=n$. Then $A$ is an $R$-subalgebra of $\mathrm{M}_{n}(R)$ and $e \in A$ is an idempotent. Also, $e A e \cong R$ as rings and $A e$ is a free $R$-module of rank $n$. It is not difficult to see that $\mu: A e \otimes_{e A e} e A \rightarrow A$ is monic and $\gamma: A \rightarrow \operatorname{End}_{e A e}(A e)$ is an injective ring homomorphism.

\section{Triangular matrix rings}

Throughout this section, $R$ and $S$ are rings and $M$ is an $S$ - $R$-bimodule satisfying the following conditions:

(M1) $M$ admits a projective resolution $P^{\bullet} \rightarrow M$ in Mod- $R$ with $P^{\bullet} \in$ $\mathcal{K}^{\mathrm{b}}\left(\mathcal{P}_{R}\right)$, in particular, $d=$ proj $\operatorname{dim} M_{R}<\infty$; and

(M2) $\operatorname{Ext}_{R}^{i}(M, R)=0$ for $i<d$.

Theorem 2.1. The triangular matrix rings

$$
\left(\begin{array}{cc}
S & M \\
0 & R
\end{array}\right) \quad \text { and } \quad\left(\begin{array}{cc}
R & \operatorname{Ext}_{R}^{d}(M, R) \\
0 & S
\end{array}\right)
$$

are derived equivalent to each other. 
Proof. Set

$$
A=\left(\begin{array}{cc}
S & M \\
0 & R
\end{array}\right) \quad \text { and } e=\left(\begin{array}{cc}
0 & 0 \\
0 & 1
\end{array}\right) \in A .
$$

It is not difficult to see that the conditions (E1)-(E5) in the preceding section are satisfied. Note also that $e A e \cong R$ and $A / A e A \cong S$ as rings. Thus we have only to show that $\operatorname{Ext}_{A}^{d+1}(A / A e A, e A) \cong \operatorname{Ext}_{R}^{d}(M, R)$.

Recall that a module $X \in \operatorname{Mod}-A$ is given by a triple $\left(X_{1}, X_{2}, \phi\right)$ of $X_{1} \in$ Mod- $S, X_{2} \in \operatorname{Mod}-R$ and $\phi \in \operatorname{Hom}_{R}\left(X_{1} \otimes_{S} M, X_{2}\right)$ and for modules $X=$ $\left(X_{1}, X_{2}, \phi\right)$ and $Y=\left(Y_{1}, Y_{2}, \psi\right)$ a homomorphism $f \in \operatorname{Hom}_{A}(X, Y)$ is given by a pair $\left(f_{1}, f_{2}\right)$ of $f_{1} \in \operatorname{Hom}_{S}\left(X_{1}, Y_{1}\right)$ and $f_{2} \in \operatorname{Hom}_{R}\left(X_{2}, Y_{2}\right)$ such that $f_{2} \circ \phi=$ $\psi \circ\left(f_{1} \otimes_{S} M\right)$ (see e.g. [?] for details). We may assume $P^{i}=0$ unless $-d \leq i \leq 0$. Since $A / A e A=(S, 0,0)$, we have a projective resolution $Q^{\bullet} \rightarrow A / A e A$ in Mod- $A$ such that $Q^{i}=\left(0, P^{i+1}, 0\right)$ for $i \neq 0$ and $Q^{0}=\left(S, M, \operatorname{id}_{M}\right)$, where $\operatorname{id}_{M}$ denotes the canonical isomorphism $S \otimes_{S} M \stackrel{\sim}{\rightarrow} M$. Also, since $e A=(0, R, 0)$, it follows that $\operatorname{Hom}_{A}^{\bullet}\left(Q^{\bullet}, e A\right) \cong \operatorname{Hom}_{R}^{\bullet}\left(P^{\bullet}, R\right)[-1]$ and hence

$$
\begin{aligned}
\operatorname{Ext}_{A}^{d+1}(A / A e A, e A) & \cong \mathrm{H}^{d+1}\left(\operatorname{Hom}_{A}^{\bullet}\left(Q^{\bullet}, e A\right)\right) \\
& \cong \mathrm{H}^{d+1}\left(\operatorname{Hom}_{R}^{\bullet}\left(P^{\bullet}, R\right)[-1]\right) \\
& \cong \mathrm{H}^{d}\left(\operatorname{Hom}_{R}^{\bullet}\left(P^{\bullet}, R\right)\right) \\
& \cong \operatorname{Ext}_{R}^{d}(M, R) .
\end{aligned}
$$

Remark 2.2. Denote by $\sigma: S \rightarrow \operatorname{End}_{R}(M), a \mapsto(x \mapsto a x)$ the ring homomorphism given by the left multiplication. Assume that $\sigma$ is injective and that if $d>0$ then $\sigma$ is an isomorphism and $\operatorname{Ext}_{R}^{i}(M, M)=0$ for $1 \leq i<d$. Then $\operatorname{Ext}_{A}^{i}(A / A e A, A)=0$ for $i \neq d+1$.

Proof. Since $Q^{\bullet}$ is a projective resolution of $A / A e A$, and since $\operatorname{Hom}_{A}^{\bullet}\left(Q^{\bullet}, e A\right) \cong$ $\operatorname{Hom}_{R}^{\bullet}\left(P^{\bullet}, R\right)[-1]$, by (M2) we have $\operatorname{Ext}_{A}^{i}(A / A e A, e A) \cong \operatorname{Ext}_{R}^{i-1}(M, R)=0$ for $i \neq d+1$. Also, since $(1-e) A=\left(S, M, \mathrm{id}_{M}\right)$, it is not difficult to see that $\operatorname{Hom}_{A}^{\bullet}\left(Q^{\bullet},(1-e) A\right)$ is isomorphic to the $(-1)$-shift of the mapping cone of the composite

$$
S \stackrel{\sigma}{\rightarrow} \operatorname{End}_{R}(M) \rightarrow \operatorname{Hom}_{R}^{\bullet}\left(P^{\bullet}, M\right) .
$$

Thus by the assumption we have $\operatorname{Ext}_{A}^{i}(A / A e A,(1-e) A)=0$ for $i \neq d+1$.

Remark 2.3. Consider the case where $R$ is a finite dimensional algebra over a field $k$ and $S=k$. By Theorem ??

$$
\left(\begin{array}{cc}
k & M \\
0 & R
\end{array}\right) \text { and }\left(\begin{array}{cc}
R & \operatorname{Ext}_{R}^{d}(M, R) \\
0 & k
\end{array}\right)
$$

are derived equivalent. Also, since $\operatorname{Ext}_{R}^{d}(M, R)$ is a finite dimensional $k$-vector space, it follows again by Theorem ?? that

$$
\left(\begin{array}{cc}
R & \operatorname{Ext}_{R}^{d}(M, R) \\
0 & k
\end{array}\right) \text { and } \quad\left(\begin{array}{cc}
k & D \operatorname{Ext}_{R}^{d}(M, R) \\
0 & R
\end{array}\right)
$$


are derived equivalent, where $D=\operatorname{Hom}_{k}(-, k)$. Thus

$$
\left(\begin{array}{cc}
k & M \\
0 & R
\end{array}\right) \text { and }\left(\begin{array}{cc}
k & D \operatorname{Ext}_{R}^{d}(M, R) \\
0 & R
\end{array}\right)
$$

are derived equivalent, which is a consequence of [?, Corollary 5.4] (see also [?]) if $\operatorname{inj} \operatorname{dim}{ }_{R} R=\operatorname{inj} \operatorname{dim} R_{R}<\infty$, since the algebras above are trivial extensions of $\Lambda=k \times R$ by $M$ and $D \operatorname{Ext}_{R}^{d}(M, R)$, respectively (see [?]), since $M \otimes{ }_{\Lambda}^{\mathbf{L}} D \Lambda[-d] \cong M \otimes{ }_{R}^{\mathbf{L}} D R[-d] \cong \operatorname{Tor}_{d}^{R}(M, D R) \cong D \operatorname{Ext}_{R}^{d}(M, R) \operatorname{in} \mathcal{D}(\operatorname{Mod}-\Lambda)$, and since $D \Lambda \in \operatorname{Mod}-\Lambda$ is a tilting module with $\Lambda \cong \operatorname{End}_{\Lambda}(D \Lambda)$ if inj $\operatorname{dim}_{R} R=$ $\operatorname{inj} \operatorname{dim} R_{R}<\infty$ (see e.g. [?, Proposition 1.6]).

\section{References}

[1] H. Abe and M. Hoshino, Derived equivalences and Gorenstein algebras, J. Pure and Appl. Algebra, 211 (2007), 55-69.

[2] M. Auslander, M. I. Platzeck and I. Reiten, Coxeter functors without diagrams, Trans. Amer. Math. Soc., 250 (1979), 1-46.

[3] M. Barot and H. Lenzing, One-point extensions and derived equivalences, J. Algebra 264 (2003), no. 1, 1-5.

[4] I. N. Bernstein, I. M. Gelfand and V. A. Ponomarev, Coxeter functors and Gabriel's theorem, Uspechi Mat. Nauk., 28 (1973), 19-38 = Russian Math. Surveys 28 (1973), 17-32.

[5] S. Brenner and M. C. R. Butler, Generalizations of the BernsteinGelfand-Ponomarev reflection functors, in: Representation theory II, 103-169, Lecture Notes in Math., 832, Springer, 1980.

[6] R. M. Fossum, Ph. A. Griffith and I. Reiten, Trivial extensions of abelian categories, Lecture Notes in Math., 456, Springer, 1976.

[7] D. Happel and C. M. Ringel, Tilted algebras, Trans. Amer. Math. Soc., 274 (1982), no. 2, 399-443.

[8] R. Hartshorne, Residues and duality, Lecture Notes in Math., 20, Springer, Berlin, 1966.

[9] Y. Miyashita, Tilting modules of finite projective dimension, Math. Z. 193 (1986), no. 1, 113-146.

[10] J. Rickard, Morita theory for derived categories, J. London Math. Soc. (2) 39 (1989), no. 3, 436-456.

[11] J. Rickard, Derived equivalences as derived functors, J. London Math. Soc. (2) 43 (1991), no. 1, 37-48. 
[12] J. L. Verdier, Catégories dérivées, état 0, in: Cohomologie étale, 262311, Lecture Notes in Math., 569, Springer, Berlin, 1977.

Institute of Mathematics, University of Tsukuba, Ibaraki, 305-8571, Japan E-mail address: abeh@math.tsukuba.ac.jp

Institute of Mathematics, University of Tsukuba, Ibaraki, 305-8571, Japan E-mail address: hoshino@math.tsukuba.ac.jp 